\title{
Status Epilepticus in Infants and Young Children Treated with Parenteral Diazepam
}

\author{
SHEILAH MCMORRIS* and P. K. A. MCWILLIAM \\ From Wakefield and Dewsbury Hospitals and Pontefract General Infirmary, Yorks.
}

Status epilepticus, meaning either continuous convulsions with unconsciousness or serial epileptic attacks, between which there is no return to consciousness, is always a serious and potentially fatal emergency. It is of special importance in paediatrics, for infants and young children have a lower convulsive theshold than adults, and recent work (Ounsted, Lindsay, and Norman, 1966) has indicated a possible relation between infantile status epilepticus and the subsequent development of temporal lobe epilepsy.

The use of parenteral diazepam in the treatment of status epilepticus, serial epilepsy, or prolonged seizure activity has been described in several communications, mostly in adults (Naquet et al., 1965; Gastaut et al., 1965; J. C. Bowe, 1965, personal communication; Bowe, 1968; Lombroso, 1966; Gordon, 1966; Prensky et al., 1967; Wilson, 1968; Parsonage and Norris, 1967; Bell, 1969), and we report here the results of treatment with this drug parenterally in 37 infants and young children during the period from September 1965 to November 1968.

\section{Patients and Treatment}

There were 20 boys and 17 girls with a mean age of $2 \cdot 6$ years (range: less than 1 day to 11 years 9 months).

The coexisting conditions and the clinical features and details of treatment are listed in the Table.

Diazepam for parenteral use is available in $2 \mathrm{ml}$. ampoules containing $10 \mathrm{mg}$., and though the intravenous route is to be preferred, this was not possible in many of our patients because of the lack of available veins in small babies or the vigorous nature of the convulsive movements or both. In these cases, the drug was administered by deep intramuscular injection. In general the effective dose varied between $0.25 \mathrm{mg}$. and $0.5 \mathrm{mg}$. per kg., but one patient required $1.4 \mathrm{mg}$. $/ \mathrm{kg}$.

Where intravenous injection was possible, this was carried out very slowly over a period of $20-60$ seconds,

Received March 7, 1969.

^Present address: Royal Hospital for Sick Children, Glasgow C.4. especially if parenteral anticonvulsants had been administered to the child before admission to hospital.

The dosages of diazepam employed, especially in the early cases, were low while experience in the use of the drug was being acquired. Hence, it seems reasonable to assume that Cases 2, 3, 10, 19, 24, 25, 29, 31, and 32 (second admission) would have been controlled earlier had a larger dose of diazepam been given initially.

In 18 cases, however (Cases 1, 4, 5, 6, 8, 9, 12, 13, 14, $18,21,22,26,27,30,35,36$, and 37$)$, lasting control of the convulsions was obtained in a mean time of 5.9 minutes (range: $10 \mathrm{sec}$. to $15 \mathrm{~min}$.) after a single injection of diazepam.

In 8 cases (Cases $3,11,15,16,17,20,23$, and 31) other anticonvulsants (usually parenteral phenobarbitone or paraldehyde) had been administered before admission and before diazepam was given, so that though it is not justifiable to credit the diazepam alone with the control of the convulsions, it was not until the diazepam was administered that control was obtained. Similarly, though Case 33 received $15 \mathrm{mg}$. intramuscular soluble phenobarbitone between the two injections of diazepam, it seems likely that the latter was responsible for the control of the seizures.

Eight deaths occurred in the series (Cases 8, 12, 20, $25,26,28,30$, and 34 ). Cases 8 and 30 were babies, aged 4 and 6 months, respectively, who had fulminating bronchopneumonia, in whom the prognosis was very bad and who might conceivably have died earlier, had their convulsions not been controlled. Case 12 had had five operations for the treatment of meconium ileus, each with a general anaesthetic. A period of apnoea followed cardiac arrest after the last of these procedures, and though the subsequent episode of status epilepticus was quickly controlled the chances of survival were poor.

Case 20 obviously had a gross degree of cerebral damage, and though the convulsions were controlled quickly, she died without regaining consciousness, and unfortunately permission for necropsy was refused. She remained unconscious and pyrexial for 64 hours after the cessation of the convulsions, so that, despite her having had parenteral phenobarbitone as well as the diazepam, there was no evidence of respiratory depression.

At necropsy Case 25 was shown to have died from 
TABLE I

Details of Patients Treated with Diazepam

\begin{tabular}{|c|c|c|c|c|c|c|c|c|c|c|}
\hline \multirow[b]{2}{*}{$\begin{array}{l}\text { Case } \\
\text { No. }\end{array}$} & \multirow[b]{2}{*}{$\begin{array}{l}\text { Age } \\
\text { (yr.) }\end{array}$} & \multirow[b]{2}{*}{ Sex } & \multirow[b]{2}{*}{$\begin{array}{l}\text { Weight } \\
\text { (kg.) }\end{array}$} & \multirow[b]{2}{*}{$\begin{array}{c}\text { Presenting } \\
\text { Clinical Features }\end{array}$} & \multicolumn{3}{|c|}{ Treatment with Diazepam } & \multirow[b]{2}{*}{$\begin{array}{c}\text { Drugs } \\
\text { Before } \\
\text { Diazepam }\end{array}$} & \multirow[b]{2}{*}{$\begin{array}{l}\text { Drugs } \\
\text { After Con- } \\
\text { trol of Sta- } \\
\text { tus }\end{array}$} & \multirow[b]{2}{*}{ Comments } \\
\hline & & & & & $\begin{array}{l}\text { First } \\
\text { Dose }\end{array}$ & $\begin{array}{c}\text { Succeeding } \\
\text { Doses in } \\
24 \mathrm{hr} .\end{array}$ & Effect & & & \\
\hline 1 & 1 & $\mathbf{M}$ & $6 \cdot 75$ & $\begin{array}{l}\text { Deeply unconscious, } \\
\text { generalized convul- } \\
\text { sions for } 2 \mathrm{hr} \text {. }\end{array}$ & $\begin{array}{l}2.5 \mathrm{mg} . \\
\text { I.M. }\end{array}$ & Nil & $\begin{array}{l}\text { Control } \\
\text { within } \\
10 \mathrm{~min} .\end{array}$ & Nil & $\begin{array}{l}\text { Pheno- } \\
\text { barb.; } \\
\text { penicillin }\end{array}$ & $\begin{array}{l}\text { URTI with bilateral } \\
\text { otorrhoea; CSF } \\
\text { normal }\end{array}$ \\
\hline 2 & 1 & F & 9 & $\begin{array}{l}\text { Microcephaly and pre- } \\
\text { vious fits; severe } \\
\text { gastro-enteritis; status } \\
\text { epilepticus for } 25 \text { min. }\end{array}$ & $\begin{array}{l}2.5 \mathrm{mg} . \\
\text { I.V. }\end{array}$ & $\begin{array}{l}2.5 \text { mg. } \\
\text { I.V. after } \\
5 \text { min. re- } \\
\text { peated on } \\
3 \text { occasions } \\
\text { in } 7 \text { hours }\end{array}$ & $\begin{array}{l}\text { Control } \\
\text { after 5th } \\
\text { injection }\end{array}$ & Nil & $\mid \begin{array}{c}\text { Oral pri- } \\
\text { midone; } \\
\text { antibiotics; } \\
\text { fluids }\end{array}$ & $\begin{array}{l}\text { Microcephaly, fits, } \\
\text { and gross mental } \\
\text { retardation }\end{array}$ \\
\hline 3 & $1 \frac{9}{4}$ & $\mathbf{F}$ & $10 \cdot 8$ & $\begin{array}{l}\text { Sudden onset of status } \\
\text { for } 50 \text { min. } 1 \text { mth. } \\
\text { after onset severe } \\
\text { measles; settled } 15 \\
\text { min. after paraldehyde } \\
\text { but recurred; diaze- } \\
\text { pam given after } 35 \\
\text { min. }\end{array}$ & $\begin{array}{l}2.5 \mathrm{mg} . \\
\text { I.M. }\end{array}$ & Nil & $\begin{array}{l}\text { Control } \\
\text { within } \\
25 \text { min. }\end{array}$ & $\begin{array}{l}\text { Paralde- } \\
\text { hyde } 1 \mathrm{ml} . \\
\text { I.M. }\end{array}$ & $\begin{array}{l}\text { Syrup } \\
\text { triclofos }\end{array}$ & $\begin{array}{l}\text { No measles ence- } \\
\text { phalitis; EEG } \\
\text { grossly abnormal; } \\
\text { no fits since; } \\
\text { mentally normal; } \\
\text { adopted child }\end{array}$ \\
\hline 4 & $10 / 12$ & $\mathbf{M}$ & $7 \cdot 65$ & $\begin{array}{l}\text { Meningococcal menin- } \\
\text { gitis; status epilepticus } \\
\text { for } 40 \text { min. }\end{array}$ & $\begin{array}{l}2.5 \mathrm{mg} . \\
\text { I.M. }\end{array}$ & Nil & $\begin{array}{l}\text { Control } \\
\text { within } \\
15 \text { min. }\end{array}$ & Nil & $\begin{array}{l}\text { Antibiotics; } \\
\text { phenobarb. }\end{array}$ & $\begin{array}{l}\text { Good progress; no } \\
\text { recurrence fits }\end{array}$ \\
\hline 5 & $3 / 12$ & $\mathbf{F}$ & $4 \cdot 05$ & $\begin{array}{l}\text { H. influenzae menin- } \\
\text { gitis; status epilepticus } \\
\text { for } 45 \mathrm{~min} \text {. }\end{array}$ & $\begin{array}{l}2 \cdot 5 \mathrm{mg} . \\
\text { I.M. }\end{array}$ & Nil & $\begin{array}{l}\text { Control } \\
\text { within } \\
5 \mathrm{~min} \text {. }\end{array}$ & Nil & $\begin{array}{c}\text { Antibiotics; } \\
\text { phenobarb. }\end{array}$ & $\begin{array}{l}\text { Good progress; no } \\
\text { further attacks }\end{array}$ \\
\hline 6 & 9 & $\mathbf{F}$ & $25 \cdot 2$ & $\begin{array}{l}\text { Epileptic; } 2 \text { previous } \\
\text { episodes of status, } \\
\text { lasting } 7 \text { and } 1 \frac{1}{2} \mathrm{hr} \text {.; } \\
\text { admitted in status of } \\
45 \text { min. duration }\end{array}$ & $\begin{array}{l}10 \mathrm{mg} . \\
\text { I.M. }\end{array}$ & Nil & $\begin{array}{l}\text { Control } \\
\text { within } \\
10 \mathrm{~min} .\end{array}$ & Nil & \begin{tabular}{|c|} 
Sulthiame; \\
chlorpro- \\
mazine; \\
primidone
\end{tabular} & Good progress \\
\hline 7 & $3 / 52$ & $\mathbf{F}$ & $2 \cdot 7$ & $\begin{array}{l}\text { Developed status epi- } \\
\text { lepticus for } 10 \mathrm{~min} \text {. } \\
\text { while under observa- } \\
\text { tion for attacks pallor, } \\
\text { rigidity, and twitching }\end{array}$ & $\begin{array}{l}2.5 \mathrm{mg} . \\
\text { I.M. }\end{array}$ & $\begin{array}{l}\text { 1.25 mg. } \\
\text { I.M. } \\
\text { 6-hourly }\end{array}$ & $\begin{array}{l}\text { Control } \\
\text { within } \\
2 \text { min. }\end{array}$ & Nil & $\begin{array}{l}\text { 1. } 25 \mathrm{mg} . ; \\
\text { diazepam } \\
\text { I.M. } \\
\text { 6-hourly } \\
\text { for } 3 \text { days }\end{array}$ & $\begin{array}{l}11 \text { days after diaze- } \\
\text { pam stopped, had } 3 \\
\text { generalized short } \\
\text { convulsions; good } \\
\text { progress since then; } \\
\text { NB prophylactic } \\
\text { use diazepam }\end{array}$ \\
\hline 8 & $4 / 12$ & $\mathbf{F}$ & $4 \cdot 95$ & $\begin{array}{l}\text { Fulminating broncho- } \\
\text { pneumonia; developed } \\
\text { severe status epilepticus } \\
\text { for } 20 \text { min. }\end{array}$ & $\begin{array}{l}2.5 \mathrm{mg} . \\
\text { I.M. }\end{array}$ & Nil & $\begin{array}{c}\text { Control } \\
\text { within } \\
10 \mathrm{~min} .\end{array}$ & Nil & $\begin{array}{l}\text { Antibiotics; } \\
\text { steroids }\end{array}$ & $\begin{array}{l}\text { Died } 4 \mathrm{hr} \text {. after } \\
\text { admission from } \\
\text { bronchopneumonia }\end{array}$ \\
\hline 9 & $1 \frac{1}{2}$ & $\mathbf{M}$ & 9 & $\begin{array}{l}\text { Family history of epi- } \\
\text { lepsy; developed status } \\
\text { epilepticus lasting } 1 \mathrm{hr} \text {. } \\
\text { with attack of } \\
\text { tonsillitis }\end{array}$ & $\begin{array}{l}2.5 \mathrm{mg} . \\
\text { I.M. }\end{array}$ & Nil & $\begin{array}{c}\text { Control } \\
\text { within } \\
5 \mathrm{~min} .\end{array}$ & Nil & Antibiotics & CSF normal \\
\hline 10 & $4 / 12$ & $\mathbf{F}$ & $4 \cdot 5$ & $\begin{array}{l}\text { Meningococcal menin- } \\
\text { gitis; severe status } \\
\text { epilepticus } 1 \frac{1}{\mathrm{hr}} \text {. }\end{array}$ & $\begin{array}{l}1 \cdot 25 \mathrm{mg} . \\
\text { I.M. }\end{array}$ & $\begin{array}{l}1 \cdot 25 \mathrm{mg} . \\
\text { after } 13 \\
\text { min. } \cdot \text { fol- } \\
\text { lowed by } \\
1 \cdot 25 \mathrm{mg} . \\
\text { after } 13 \\
\text { min. }\end{array}$ & $\begin{array}{c}\text { Control } \\
7 \text { min. after } \\
\text { 3rd injec- } \\
\text { tion }\end{array}$ & Nil & $\begin{array}{c}\text { Antibiotics; } \\
\text { barbitu- } \\
\text { rates }\end{array}$ & $\begin{array}{l}\text { 3-day history crying } \\
\text { before onset status }\end{array}$ \\
\hline 11 & $2 \frac{1}{2}$ & $\mathbf{M}$ & $16 \cdot 2$ & $\begin{array}{l}\text { Status epilepticus } 2 \mathrm{hr} \\
\text { no response to I.M. } \\
\text { phenobarb. }\end{array}$ & $\begin{array}{l}10 \mathrm{mg} . \\
\text { I.M. }\end{array}$ & Nil & $\begin{array}{l}\text { Control } \\
\text { after } 3 \\
\text { min. }\end{array}$ & $\begin{array}{l}\text { Phenobarb. } \\
30 \mathrm{mg} .1 \\
\text { hr. } 40 \mathrm{~min} . \\
\text { after onset } \\
\text { of status }\end{array}$ & Phenobarb. & $\begin{array}{l}\text { Transient rt. hemi- } \\
\text { paresis; abnormal } \\
\text { EEG (post-ictal) }\end{array}$ \\
\hline
\end{tabular}


TABLE I-continued

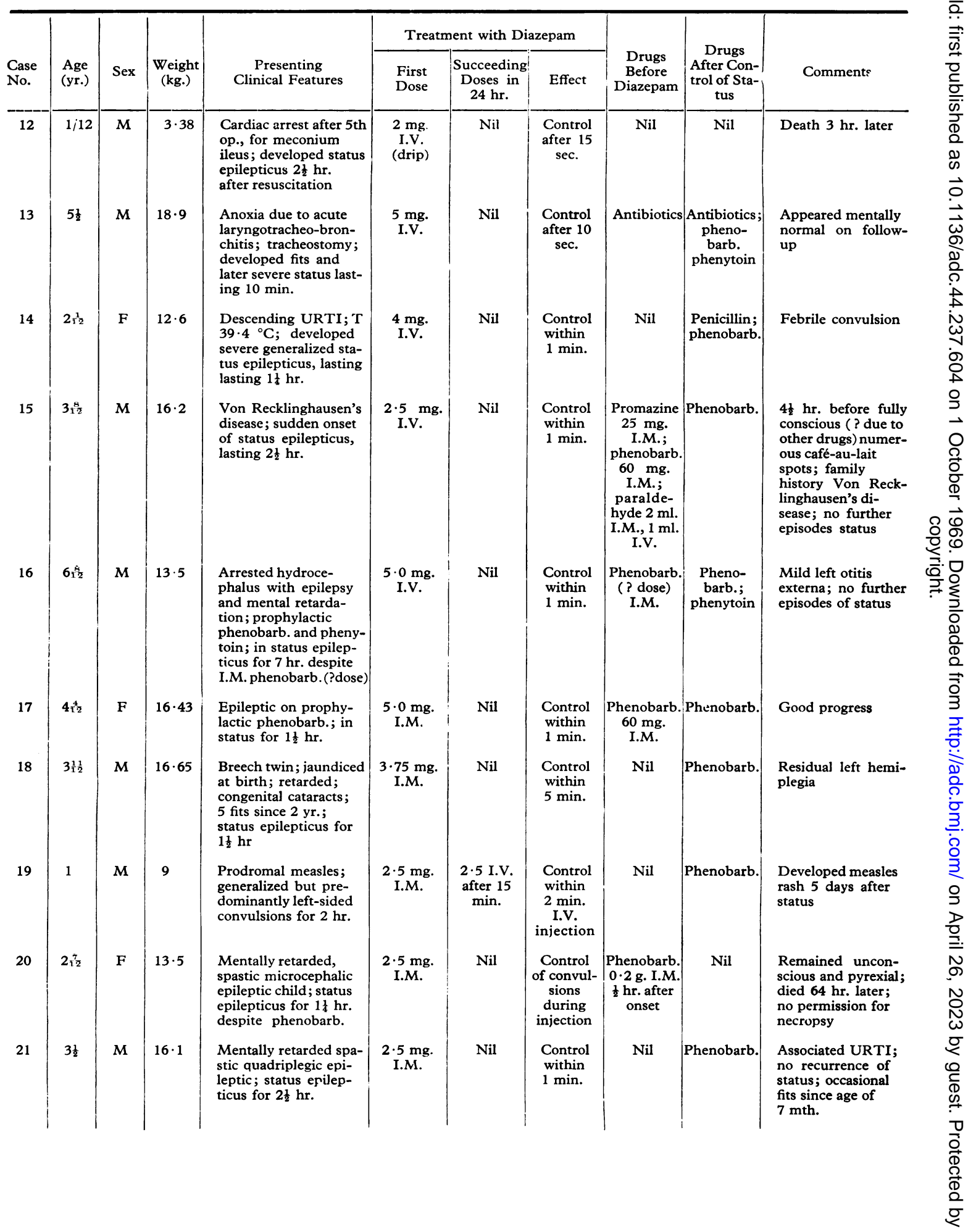


TABLE I-continued

\begin{tabular}{|c|c|c|c|c|c|c|c|c|c|c|}
\hline \multirow[b]{2}{*}{$\begin{array}{l}\text { Case } \\
\text { No. }\end{array}$} & \multirow[b]{2}{*}{$\begin{array}{l}\text { Age } \\
\text { (yr.) }\end{array}$} & \multirow[b]{2}{*}{ Sex } & \multirow[b]{2}{*}{$\begin{array}{l}\text { Weight } \\
\text { (kg.) }\end{array}$} & \multirow[b]{2}{*}{$\begin{array}{c}\text { Presenting } \\
\text { Clinical Features }\end{array}$} & \multicolumn{3}{|c|}{ Treatment with Diazepam } & \multirow[b]{2}{*}{$\begin{array}{c}\text { Drugs } \\
\text { Before } \\
\text { Diazepam }\end{array}$} & \multirow{2}{*}{$\begin{array}{l}\text { Drugs } \\
\text { After Con- } \\
\text { trol of Sta- } \\
\text { tus }\end{array}$} & \multirow[b]{2}{*}{ Comments } \\
\hline & & & & & $\begin{array}{l}\text { First } \\
\text { Dose }\end{array}$ & $\begin{array}{c}\text { Succeeding } \\
\text { Doses in } \\
24 \mathrm{hr} .\end{array}$ & Effect & & & \\
\hline 22 & $6 \frac{1}{12}$ & $\mathbf{F}$ & 18 & $\begin{array}{l}\text { Spina bifida, hydro- } \\
\text { cephalus; meningocele } \\
\text { and paraplegia; Spitz } \\
\text { Holter valve, genera- } \\
\text { lized convulsions for } \\
\frac{1}{2} \mathrm{hr} \text {. }\end{array}$ & $\begin{array}{l}5 \text { mg. } \\
\text { I.V. }\end{array}$ & Nil & $\begin{array}{l}\text { Control } \\
\text { within } \\
1 \mathrm{~min} .\end{array}$ & Nil & Phenobarb. & $\begin{array}{l}\text { Admitted for gene- } \\
\text { ral care during } \\
\text { mother's illness; } \\
\text { one previous epi- } \\
\text { sode of status }\end{array}$ \\
\hline 23 & $3 \frac{1}{1} \frac{1}{2}$ & $\mathbf{M}$ & $17 \cdot 1$ & $\begin{array}{l}\text { 1-yr. history monthly } \\
\text { fits; prophylactic } \\
\text { phenobarb. and pheny- } \\
\text { toin; admitted after } 4 \\
\text { major fits in } 24 \text { hr.; } \\
\text { developed severe status } \\
\text { epilepticus soon after } \\
\text { admission lasting } 10 \\
\text { min. }\end{array}$ & $\begin{array}{l}5 \cdot 0 \mathrm{mg} . \\
\text { I.V. }\end{array}$ & Nil & $\begin{array}{l}\text { Complete } \\
\text { control } \\
\text { within } \\
4 \text { min.; } \\
\text { slowing } \\
\text { of convul- } \\
\text { sions after } \\
2 \text { min. }\end{array}$ & $\begin{array}{c}\text { Phenobarb. } \\
\text { phenytoin } \\
\text { orally just } \\
\text { before on- } \\
\text { set of sta- } \\
\text { tus }\end{array}$ & $\begin{array}{l}\text { Phenobarb. } \\
\text { phenytoin }\end{array}$ & No further status \\
\hline 24 & $2 \frac{3}{12}$ & $\mathbf{F}$ & $13 \cdot 05$ & $\begin{array}{l}\text { URTI; left otitis media; } \\
\text { status epilepticus of } \\
2 \mathrm{hr} \text {. duration }\end{array}$ & $\begin{array}{l}2.5 \mathrm{mg} \text {. } \\
\text { I.M. }\end{array}$ & $\begin{array}{l}2.5 \mathrm{mg} . \\
\text { I.M. } \\
\text { after } 15 \\
\text { min. }\end{array}$ & $\begin{array}{l}\text { Control } \\
10 \text { min. } \\
\text { after 2nd } \\
\text { inject. }\end{array}$ & Nil & $\begin{array}{c}\text { Pheno- } \\
\text { barb.; } \\
\text { antibiotics }\end{array}$ & No further status \\
\hline 25 & 1 day & $\mathbf{M}$ & $2 \cdot 14$ & $\begin{array}{l}\text { Collapsed, apnoeic and } \\
\text { cardiac arrest after } \\
\text { traumatic breech deli- } \\
\text { very; resuscitated; } \\
\text { developed serial fits } \\
\text { lasting } 5 \text { hr.; } \\
\text { unconscious }\end{array}$ & $\begin{array}{l}1 \mathrm{mg} . \\
\text { I.M. }\end{array}$ & Nil & $\begin{array}{l}\text { No more } \\
\text { fits for } 1 \frac{1}{\text { hr., then }} \\
\text { occasional } \\
\text { twitching }\end{array}$ & Nil & $\begin{array}{l}\text { Phenobarb. } \\
7.5 \text { mg. } 3- \\
\text { hourly; } \\
\text { phenytoin, } \\
15 \text { mg. } \\
\text { I.M. } \\
3 \text { hourly }\end{array}$ & $\begin{array}{l}\text { Died same day; } \\
\text { PM: bilateral } \\
\text { tentorial tears }\end{array}$ \\
\hline 26 & 1 day & $\mathbf{M}$ & $2 \cdot 59$ & $\begin{array}{l}\text { LSCS; cord trapped } \\
\text { beside head; cried } 10 \\
\text { min. after birth (intu- } \\
\text { bated); generalized } \\
\text { convulsions } 15 \mathrm{hr} \text {. } \\
\text { after birth for } 10 \mathrm{~min} \text {. }\end{array}$ & $\begin{array}{l}1 \mathrm{mg} . \\
\text { I.M. }\end{array}$ & Nil & $\begin{array}{l}\text { Control } \\
\text { within } \\
5 \text { min. }\end{array}$ & Nil & Nil & $\begin{array}{l}\text { Remained limp and } \\
\text { hypotonic; died } \\
\text { despite further re- } \\
\text { suscitation; PM: } \\
\text { anoxaemia due to } \\
\text { atelectasis and cere- } \\
\text { bral oedema (no } \\
\text { tear) }\end{array}$ \\
\hline 27 & 1 day & F & $3 \cdot 15$ & $\begin{array}{l}\text { Irritable and cyanotic } \\
\text { attacks after cord round } \\
\text { neck twice; } 7 \frac{1}{2} \mathrm{hr} \text {. } \\
\text { after birth developed } \\
\text { rt.-sided twitching } \\
\text { lasting } 3 \mathrm{hr} \text {. }\end{array}$ & $\begin{array}{l}1 \mathrm{mg} . \\
\text { I.M. }\end{array}$ & $\begin{array}{l}1 \mathrm{mg} \text {. } \\
\text { I.M. } \\
6 \text { hourly } \\
\text { for } 24 \mathrm{hr} \text {. }\end{array}$ & $\begin{array}{c}\text { Control } \\
\text { within } \\
5 \text { min. of } \\
\text { first } \\
\text { injection }\end{array}$ & Nil & $\begin{array}{l}\text { Oral } \\
\text { chloral } \\
\text { after } \\
\text { diazepam }\end{array}$ & $\begin{array}{l}\text { Prophylactic use of } \\
\text { diazepam gradual } \\
\text { improvement; chlo- } \\
\text { ral reduced and } \\
\text { stopped after } 2 \text { dy.; } \\
\text { left CDH detected; } \\
\text { no other abnorma- } \\
\text { lity detected } 2 \text { wk. } \\
\text { later }\end{array}$ \\
\hline 28 & 8 & $\mathbf{M}$ & $22 \cdot 5$ & $\begin{array}{l}\text { Diabetic coma; no pre- } \\
\text { vious epilepsy or family } \\
\text { history of same }\end{array}$ & $\begin{array}{l}5 \text { mg. } \\
\text { I.V. }\end{array}$ & $\begin{array}{l}10 \mathrm{mg} . \\
\text { I.V. } \\
8 \frac{1}{2} \mathrm{hr} . \\
\text { later }\end{array}$ & See & text & See t & \\
\hline 29 & $2 \frac{1}{12}$ & $\mathbf{F}$ & $12 \cdot 6$ & $\begin{array}{l}\text { URTI and definite } \\
\text { urinary tract infection; } \\
\text { admitted with severe } \\
\text { status of } 7 \frac{g}{\mathrm{hr}} \text {. dura- } \\
\text { tion }\end{array}$ & $\begin{array}{l}2.5 \mathrm{mg} . \\
\text { I.V. }\end{array}$ & $\begin{array}{l}2.5 \mathrm{mg} . \\
\text { I.M. } \\
15 \mathrm{~min} . \\
\text { later } \\
2.5 \mathrm{mg} . \\
\text { I.V.; } 30 \\
\text { min. later }\end{array}$ & $\begin{array}{l}\text { Control } \\
\text { within } \\
5 \text { min. of } \\
\text { third } \\
\text { injection }\end{array}$ & Nil & $\begin{array}{c}\text { Phenobarb. } \\
\text { antibiotics }\end{array}$ & $\begin{array}{l}\text { Progressive eleva- } \\
\text { tion of conscious- } \\
\text { ness; rt.-sided } \\
\text { residual hemiplegia; } \\
\text { confirmatory (L) } \\
\text { cortical damage on } \\
\text { EEG }\end{array}$ \\
\hline 30 & $\frac{1}{2}$ & $\mathbf{M}$ & 9 & $\begin{array}{l}\text { Admitted deeply un- } \\
\text { conscious and convul- } \\
\text { sing for } 30 \text { min.; temp. } \\
41 \cdot 1{ }^{\circ} \mathrm{C} \text {; ; broncho- } \\
\text { pneumonia }\end{array}$ & $\begin{array}{l}2.5 \mathrm{mg} \text {. } \\
\text { I.M. }\end{array}$ & Nil & $\begin{array}{c}\text { Control } \\
\text { within } \\
5 \text { min. of } \\
\text { injection }\end{array}$ & $\mathrm{Nil}$ & Antibiotics & $\begin{array}{l}\text { Slight improvement } \\
\text { in conscious level, } \\
\text { then died suddenly; } \\
\text { PM: fulminating } \\
\text { bronchopneumonia }\end{array}$ \\
\hline
\end{tabular}


TABLE I-continued

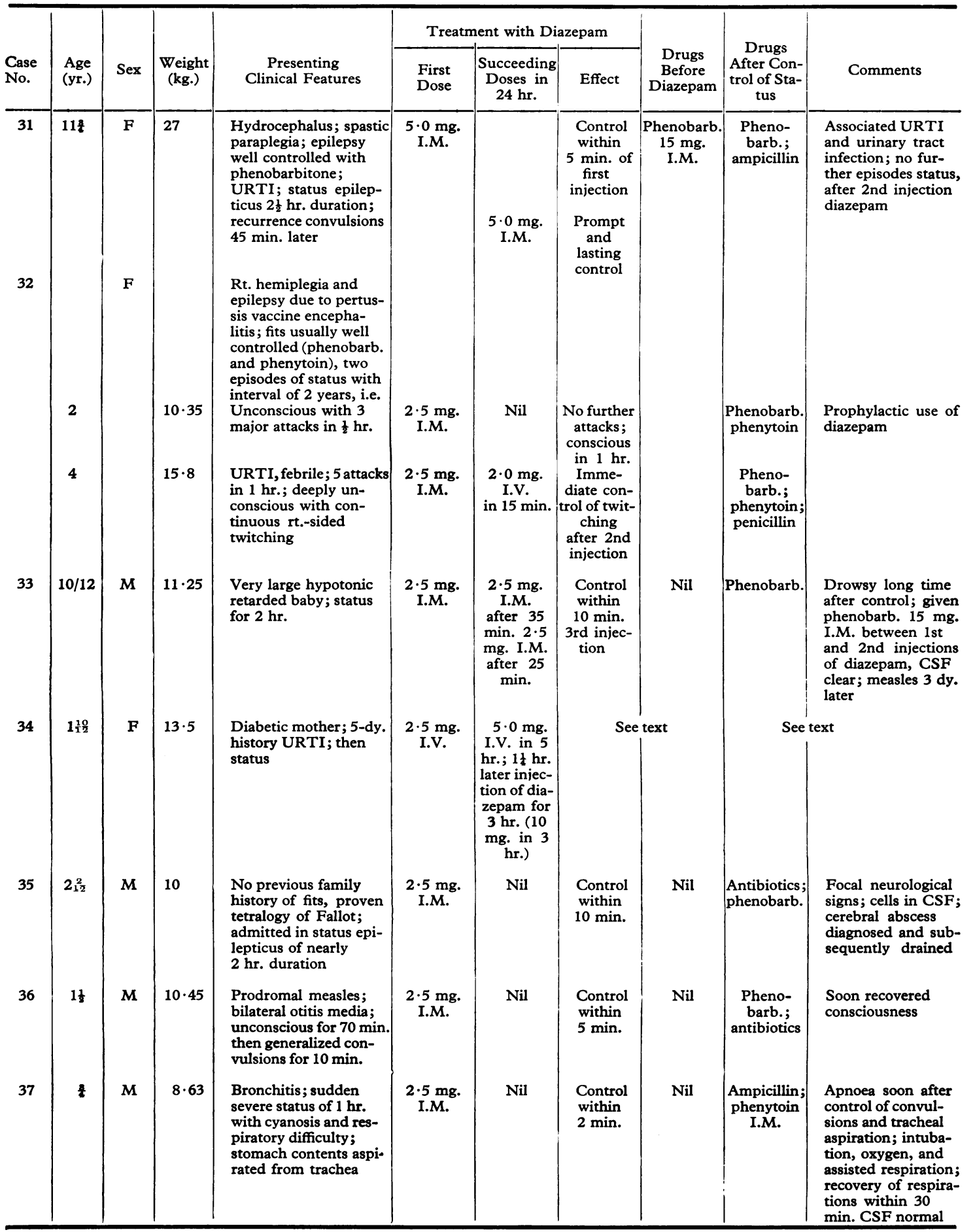




\section{Status Epilepticus in Infants and Young Children Treated with Parenteral Diazepam}

bilateral tentorial tears and Case 26 of atelectasis with cerebral oedema.

Case 28 was admitted in diabetic pre-coma (blood sugar $450 \mathrm{mg}$. $/ 100 \mathrm{ml}$.) after three weeks of typical prediabetic symptoms. After appropriate treatment his blood sugar had fallen to $160 \mathrm{mg}$. $/ 100 \mathrm{ml}$. within 6 hours, with concomitant improvement in consciousness; $4 \frac{1}{2}$ hours later he had the first of two attacks in 4 hours in which he was flushed, screaming, and incontinent of faeces, and 3 hours later he had a predominantly rightsided convulsion lasting $1 \frac{1}{2}$ minutes, at which time his blood sugar was $208 \mathrm{mg} . / 100 \mathrm{ml}$. He was rousable, and since there was no hypoglycaemia, $5 \mathrm{mg}$. diazepam was given intravenously as prophylaxis against further attacks. His general condition deteriorated despite attempts at biochemical correction, and $8 \frac{1}{2}$ hours later he had four severe major fits within 15 minutes. Again as a prophylactic measure, $10 \mathrm{mg}$. diazepam was given intravenously 45 minutes later; he coughed and suddenly developed apnoea with a cardiac triple rhythm. Resuscitative measures were adopted, but 3 hours later cardiac arrest occurred and he died. CSF, blood sugar, and blood urea estimations were within normal limits. Necropsy revealed abnormal pancreatic islet cells, flattening of cerebral convolutions, with a cerebellar pressure cone and collapse of the right lung. Virus studies were negative, and a coincidental encephalitis was reasonably excluded histologically.

Case 34 was a small girl with a diabetic mother. She was admitted to another hospital where she was noted to be collapsed, comatose, and to have clinical signs of bronchopneumonia, after having had several generalized convulsions within $1 \frac{1}{2}$ hours. A few days earlier she had had a fall from her tricycle and had developed an upper respiratory infection. She received intramuscular injections of soluble penicillin and coramine, but she remained unconscious and continuously convulsing and was transferred to Pontefract General Infirmary $7 \frac{1}{2}$ hours later. Shortly before transfer, some glycosuria was reported. On admission she was deeply unconscious and having continuous right-sided convulsions. (Blood sugar $23 \mathrm{mg} . / 100 \mathrm{ml}$., CSF sugar $17 \mathrm{mg}$. $100 \mathrm{ml}$., CSF protein and cells normal.) An intravenous drip of $50 \%$ dextrose was set up, and diazepam $2.5 \mathrm{mg}$. was given intravenously. She remained unconscious, with slow right-sided convulsive movements. 5 hours later, the blood sugar was normal and a further $5 \mathrm{mg}$. diazepam was given intravenously, again without any apparent effect. $1 \frac{1}{4}$ hours later, an infusion of diazepam and $50 \%$ dextrose was started ( $30 \mathrm{mg}$. diazepam in $600 \mathrm{ml} .50 \%$ dextrose) and ran continuously for 3 hours $(3.3 \mathrm{mg}$. $/ \mathrm{hr}$.) when the convulsions stopped, after some preliminary slowing and some improvement in the level of consciousness. 40 minutes later, her normal respirations suddenly stopped. Mechanically assisted respiration was carried out before spontaneous respiration was gradually resumed and re-established $3 \frac{1}{2}$ hours later. One hour later, she moved all her limbs normally, reacted to painful stimuli, and exhibited both corneal reflexes. She then developed signs of increased intracranial pressure and died suddenly, soon after transfer to a neurosurgical unit. Necropsy revealed slight congestion of the brain but no obvious focal lesion. Histology of the pancreas suggested a prediabetic state, with degranulation of the $\beta$ cells suggesting an irritation release of insulin into the blood-stream, thus creating prediabetic hypoglycaemic coma. Collapse of the lower lobe of the right lung and patchy atelectasis elsewhere were also noted.

\section{Discussion}

In all patients except 3 (Cases 28, 34, and 37) the anticonvulsant effect of parenteral diazepam was demonstrated without apparent complications or side-effects. Intramuscular injections were used more often than intravenous injections because of the impracticability of the latter, yet prompt and lasting control was achieved in a significant number of cases, often very quickly, as described by Naquet et al. (1965).

Cases 28 and 34 are reported in some detail owing to their similarity and because many factors about them still remain unexplained. In Case 28, it seemed reasonable to administer a second dose of diazepam after the 4 major fits in rapid succession, especially since this patient had tolerated his earlier dose of diazepam apparently well. Case 34 was also disturbing. It seems likely that her long history of coma, uncontrolled convulsions, and hypoglycaemia would have produced irreversible cerebral damage. One can but speculate on the cause of her reported glycosuria. Possibly the coramine may have been responsible or she may have had a fluctuating blood sugar before her hypoglycaemic phase.

Having regard to the necropsy findings in these two children it seems prudent at present to advise extreme caution in the use of diazepam in patients who have diabetes mellitus or disordered carbohydrate metabolism in association with status epilepticus.

The possibility of respiratory depression was always in mind, especially where barbiturates had been administered previously, because of the possible synergism between the two drugs.

Apart from Cases 28 and 34, Case 37 was the only other to cause anxiety from this aspect. Earlier on the day of admission penicillin therapy had been given for bronchitis. 5 hours later, he suddenly developed severe status epilepticus with marked cyanosis, which lasted for an hour before being rapidly controlled by diazepam. The convulsions ceased completely within 2 minutes but then apnoea quickly supervened. Further tracheal aspiration and assisted respiration (with oxygen via an endotracheal tube) were followed by the 
disappearance of the cyanosis and the resumption of spontaneous respiration within 30 minutes. He had unquestionable respiratory depression of central origin after the injection of diazepam, but it seems likely that his pre-existing hypoxic state was a significant predisposing factor.

Gastaut et al. (1965) describe shallow respiration, apnoea, and feared collapse in an infant of 9 months who had prompt control of a predominantly rightsided clonic seizure within 20 seconds of an intravenous injection of $6 \mathrm{mg}$. diazepam. Apparently there was a spontaneous return of normal respiration.

Irreversible respiratory arrest is reported by Wilson (1968) in a $2 \frac{1}{2}$-year-old boy suffering from a malignant glioma from which a recent biopsy had been taken. Status epilepticus developed which was controlled by $20 \mathrm{mg}$. intravenous diazepam, but a further episode of status epilepticus occurred 12 hours later. Intravenous diazepam (10 mg.) terminated the status but irreversible respiratory arrest followed. Since no necropsy was performed it remains conjectural as to whether the arrest was due to the diazepam or to haemorrhage into the glioma.

Prensky et al. (1967) in a series of 30 patients (20 having acute and 10 having slowly progressive cerebral disease) evaluated the effects of intravenous diazepam in the treatment of prolonged seizure activity. 2 patients in this series were children, aged 4 months and 6 years. 3 cases had respiratory depression, including the 4 monthold child; 2 cases had transient hypotension; and 1 case had fatal cardiac arrest. In 3 of their 5 cases of respiratory or vasomotor depression, including the 4-month-old child, intravenous anticonvulsants including barbiturates had been administered shortly before the diazepam. They suggest that in patients with acute cerebral disorders, who have recently received intravenous barbiturates, diazepam should be used with caution.

More recently, Bell (1969) has reported the results of the treatment of 25 patients suffering from status epilepticus transferred to a neuropsychiatric unit from psychiatric hospitals. The mean age of the patients was 39 (range 7 to 69) years, and only 3 patients were less than 20 years old. Their normal anticonvulsant medication was continued during treatment with diazepam. Intramuscular phenobarbitone and phenytoin (and in two cases, paraldehyde) were given when there was a poor or limited response to diazepam.

Respiratory depression was reported only once. It lasted about half an hour, involved CheyneStokes respiration, and followed a small dose of diazepam. The same patient, however, had no respiratory depression in a subsequent episode of status epilepticus in which considerably larger doses of diazepam were employed following parenteral barbiturates.

Hypotension was reported in 6 of the adults, severely in 2. One of the patients was the one in whom respiratory depression was reported, whose blood pressure readings were said to have been lower than any found in the regular 2 hourly recordings kept for the preceding 2 months, though the reason for these frequent and prolonged recordings is not stated.

All Bell's patients came from psychiatric hospitals and presumably were receiving other drugs, so that this may have been another factor in the production of the hypotension.

No blood pressure monitoring was undertaken in our patients, as it seems to be difficult to do in a vigorously convulsing infant or child. Parsonage and Norris (1967) report hypotension to have been noted before death in one of their cases, but having regard to the clinical details and necropsy findings, they considered that diazepam was not responsible. They further stated that in none of their 9 cases (the youngest of whom was aged 12) was there any evidence of depression of vital centres, despite the infusion of comparatively large amounts of diazepam. They did note, however, that with doses of more than $10 \mathrm{mg}$. diazepam a hypnotic effect was observed in most of their cases, which had to be considered when assessing levels of consciousness.

In this connexion it is worth noting the work of Baird and Pileggi (1968), who described depression or even abolition of the corneal reflexes for variable periods of time, ranging from 10 minutes to several hours after the intravenous administration of diazepam to children. They suggest that diazepam may have a selective action on the midbrain, and that this should be of interest to clinicians who administer intravenous diazepam. In none of our patients were we aware of this phenomenon, possibly because our dosages of diazepam were considerably smaller than those quoted by Baird and Pileggi (i.e. mean dose of $4.0 \mathrm{mg}$. $/ \mathrm{kg}$. in 35 subjects).

Other side-effects which have been reported in the past, such as ataxia (Trolle, 1965) or mental disturbances (Femi-Pearse, 1966), were not noted in any of our cases.

Diazepam for injection was found to be effective, well tolerated, and convenient to use. It is readily available, requires no reconstitution, is contained in a small volume of fluid, there is no evidence that it causes local tissue damage, the injection is relatively painless (important if used prophylacti- 
cally), and can be administered using disposable polystyrene syringes. These practical advantages, to some of which Bowe (1968) has already drawn attention, make the preparation suitable for domiciliary paediatric practice, especially as a 'first choice' drug. It assists in the prompt control of a dangerous condition though the need for evaluation of the cause still remains.

\section{Summary}

The use of parenteral diazepam in the treatment of status epilepticus in 37 infants and young children is described. In 18 cases, prompt and lasting control of the seizures followed a single (usually intramuscular) injection of the drug. In 9 cases more than one injection was required, and in 8 cases other anticonvulsants had been given, often before admission to hospital.

Eight patients died, 2 of whom had diabetes mellitus, and though there was no evidence that diazepam was responsible, caution is urged regarding the use of the drug in similar circumstances, pending further research. One case of reversible respiratory depression occurred after the use of the drug in a hypoxic infant.

Subject to the above observations it is concluded that parenteral diazepam is an effective and safe anticonvulsant for the treatment of status epilepticus in infants and young children, and the view that this is a 'first choice' drug is supported.

We thank Drs. J. D. Pickup and C. S. Livingstone for permission to treat patients under their care and for their continued interest in this investigation. We are grateful to Dr. D. G. Harriman and Dr. C. R. Abbott of the General Infirmary, Leeds, for the histological reports, and to Dr. C. G. Burgess, Roche Products Ltd., for advice and supplies of parenteral diazepam.

\section{REFERENCES}

Baird, H. W., and Pileggi, A. J. (1968). Diminished corneal reflex after diazepam. Lancet, $2,106$.

Bell, D. S. (1969). Dangers of treatment of status epilepticus with diazepam. Brit. med. F., 1, 159.

Bowe, J. C. (1968). Epilepsy: the present position in Britain. Research Review, Med. News, 23 February, 1968.

Femi-Pearse, D. (1966). Experience with diazepam in tetanus. Brit. med. F., 2, 862.

Gastaut, H., Naquet, R., Poiré, R., and Tassinari, C. A. (1965). Treatment of status epilepticus with diazepam ('Valium'). Epilepsia (Amst.), 6, 167.

Gordon, N. S. (1966). Treatment of status epilepticus with diazepam. Develop. Med. Child Neurol., 8, 668.

Lombroso, C. T. (1966). Treatment of status epilepticus with diazepam. Neurology (Minneap.), 16, 629.

Naquet, R., Soulayrol, R., Dolce, G., Tassinari, C. A., Broughton, R., and Loeb, H. (1965). First attempt at treatment of experimental status epilepticus in animals and spontaneous status epilepticus in man with diazepam. ('Valium'). (abstr.). Electroenceph. clin. Neurophysiol., 18, 427.

Ounsted, C., Lindsay, J., and Norman, R. (1966). Biological Factors in Temporal Lobe Epilepsy (Clinics in Developmental Medicine, no. 22). Spastics Society and Heinemann, London.

Parsonage, M. J., and Norris, J. W. (1967). Use of diazepam in treatment of severe convulsive status epilepticus. Brit. med. f., 3, 85 .

Prensky, A. L., Raff, M. C., Moore, M. J., and Schwab, R. S. (1967). Intravenous diazepam in the treatment of prolonged seizure activity. New Engl. F. Med., 276, 779.

Trolle, E. (1965). Diazepam ('Valium') in the treatment of epilepsy. A report of 50 cases. Acta neurol. scand., 41, Suppl. 13, 535.

Wilson, P. J. E. (1968). Treatment of status epilepticus in neurosurgical patients with diazepam ('Valium'). Brit. F. clin. Pract., 22, 21.

Correspondence to P. K. A. McWilliam, The General Infirmary, Pontefract, Yorks. 